\title{
Intradural Extramedullary Ewing Sarcoma in an Adolescent Female
}

\author{
Mikkel Kunwald (D)', Christian Lund Straszek ${ }^{2-4}$, Carsten Reidies Bjarkam ', Marianne Schmidt Ettrup ${ }^{5}$, \\ Torjus Skajaa $^{6}$, Janus Laust Thomsen $\mathbb{D}^{2}$, Mikkel Thusgaard ${ }^{7}$, Morten Zebitz Steiness ${ }^{1}$ \\ 'Department of Neurosurgery, Aalborg University Hospital, Aalborg, Denmark; ${ }^{2}$ Center for General Practice, Aalborg University, Aalborg, Denmark; \\ ${ }^{3}$ Department of Health Science and Technology, Aalborg University, Aalborg, Denmark; ${ }^{4}$ Department of Physiotherapy, University College of \\ Northern Denmark, Aalborg, Denmark; ${ }^{5}$ Department of Pathology, Aalborg University Hospital, Aalborg, Denmark; ${ }^{6}$ The Department of Paediatrics \\ and Adolescent Medicine, Aarhus University Hospital, Aarhus, Denmark; ${ }^{7}$ Family practice, Laegehuset i Hals, Hals, Denmark
}

Correspondence: Mikkel Kunwald, Department of Neurosurgery, Aalborg University Hospital, Hobrovej 18-22, Aalborg, 9000, Denmark, Tel +4597662400, Email m.kunwald@rn.dk

\begin{abstract}
A 15-year-old female experiencing lumbar pain without prior trauma. Despite being prescribed strong analgesics by her family physician, the symptoms intensified, and she was referred to diagnostic imaging. Magnetic resonance imaging revealed an intradural extramedullary tumor in the spinal canal, located behind the L3 corpora. The patient underwent neurosurgery with a complete resection of the tumor. The histopathologic examination revealed Ewing sarcoma by identifying EWSR1 gene in the extracted tissue. The patient was subsequently referred for extensive specialized oncological treatment including photon irradiation therapy and chemotherapy. Thirty-six months on from the initial diagnosis, the patient is doing well and suffers no sequelae apart from hypogonadism.
\end{abstract}

Keywords: intradural, extramedullary, Ewing sarcoma, tumor, spine, adolescent

\section{Introduction}

Among patients between 8 and 19 years of age $40 \%$ experience lumbar spinal pain. ${ }^{1}$ However, less than $1 \%$ will have a sinister underlying pathology such as fracture, infection, or cancer. ${ }^{1}$ Here, we present a clinical course for an adolescent female with lumbar spinal pain due to an intradural extramedullary Ewing sarcoma from the family physician to the departments of neurosurgery, pathology and oncology.

Signed consent for publication was obtained from both the patient and guardian. No institutional approval was required to publish the case.

\section{Pre-Hospital Course in Family Practice}

A 15-year-old female consulted her family physician due to ongoing lumbar pain radiating into the lower leg on the righthand side. The symptoms had an acute onset three weeks earlier following an abdominal exercise (the crunch exercise). No abnormalities were found during assessment of spine and neurology, and the patient had no prior history of spinerelated pain. The piriformis muscle was found to be tense, and the patient was referred to physiotherapy to relieve piriformis-induced pain.

Twenty days after the initial consultation, the condition had not improved. The patient was re-examined by the family physician and afterwards prescribed $200 \mathrm{mg}$ Ibuprofen and $500 \mathrm{mg}$ Paracetamol three times per day to reduce the pain.

Twenty-two days after the initial consultation, the patient consulted her family physician due to lack of pain remission and reported that she woke up from her sleep several times during the night due to pain from the lumbar spine. The pain was provoked when she lay in a supine position and during right-hand-side extension of the hip from a prone position. As previously, spinal and neurological functions were found to be normal. The patient was referred to intensive physiotherapy and was prescribed Codeine to supplement the analgesics prescribed two days prior. 

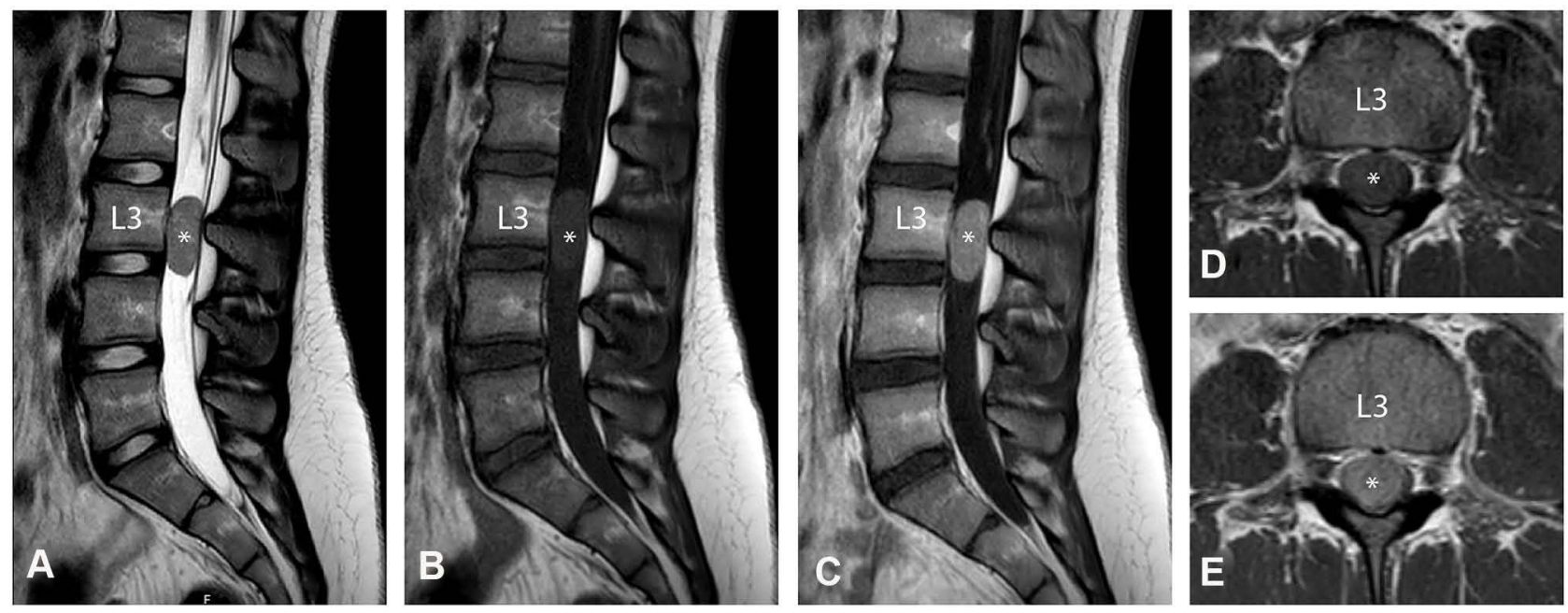

Figure I MRI-based visualization of the the tumor process $\left(^{*}\right)$ located in the lumbar spinal canal behind the third lumbar vertebral body (L3). (A) Mid-sagittal T2-weighted MRI, (B) Mid-sagittal TI-weighted MRI, (C) Mid-sagittal TI-weighted MRI with contrast (Clariscan), (D) Axial TI-weighted MRI, (E) Axial TI-weighted MRI with contrast (Clariscan).

Twenty-seven days after the initial consultation, the patient consulted her family physician due to increased pain symptoms. She reported periodic sensations of stabbing pain, and she could not contribute sufficiently to the assessment of spinal and neurological function. She was admitted to a local hospital due to a lack of remission for lumbar spinal pain despite extensive analgesic coverage.

\section{MR Imaging}

Upon admission, magnetic resonance imaging (MRI) was performed revealing an intradural, extramedullary tumor in the spinal canal located behind the L3 corpora measuring $33 \times 17 \times 13 \mathrm{~mm}$ (Figure 1). It seemed to be adjacent to a single traversing nerve root. The findings and the clinical symptoms of radiating pain in one leg seemed most likely to represent a benign tumor, eg a schwannoma or ependymoma.

\section{Neurosurgical Procedure}

Gross total removal of the tumor was performed via a posterior approach. During surgery, the lamina and spinous process of the L2, L3 and L4 were removed en bloc to be re-attached at the end of the procedure (laminoplasty). The remains of the flavous ligaments were removed and the dura exposed. Upon dural opening there was a flush of liquor under pressure. Subsequently, a dark and hemorrhagic tumor was identified. Under microscopic magnification microsurgical dissection and freeing of the traversing nerve roots was performed. The tumor was found to be adherent to the filum terminale but was free-dissected without cutting the filament. The tumor was more adherent to the traversing nerve root than would be expected from a schwannoma. Ultimately, a complete resection of the tumor was obtained, and the removed tissue was dispatched for histopathologic examination. The patient was discharged from the surgical unit after a few days, in good condition and with normal neurology.

Fourteen days post surgery, after the result of the histopathological examination, a PET-CT scan was performed. No sign of residual disease in the spine nor signs of metastases was found on the scan.

\section{Pathology}

Histopathological examination of formalin-fixed paraffin-embedded tissue showed a tumor composed of uniform small round cells with finely dispersed chromatin without nucleoli and only sparse clear cytoplasm (Figure 2A). The neoplastic cells formed sheets and scant noduli with intervening fibrovascular bands (Figure 2A). There was dispersed mitosis. There were no obvious formations of Homer Wright rosettes. There was alternating foci of necrosis (Figure 2A). Immunohistochemistry showed a positive stain in vimentin, S-100 and strong membranous stain in CD99. There was focally stained in synaptophysin 

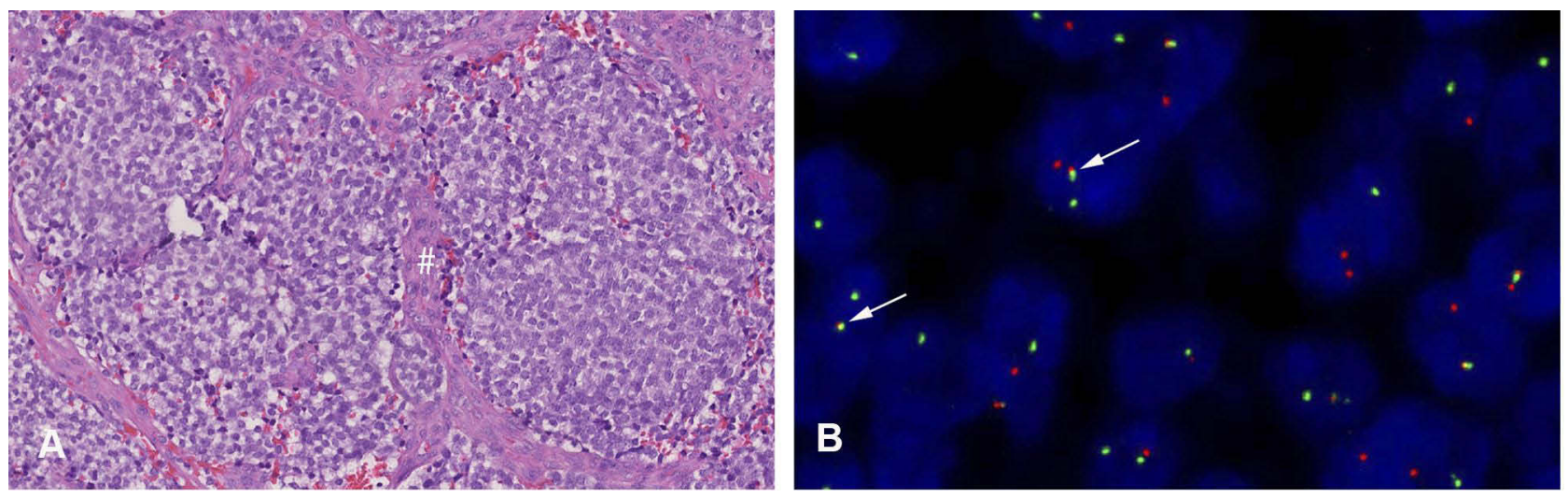

Figure 2 Tumor pathology. (A) Hematoxylin and eosin (HE) stain x20 magnification, demonstrating uniform small round cells with fine dispersed chromatin with only sparse cytoplasm. The tumor cells are arranged in noduli with intervening lighter fibrovascular bands $\left({ }^{\#}\right)$. (B) Fluorescence In Situ Hybridization (FISH). Dual color break apart probe with the red fluorochrome direct proximal of the EWSRI gene while the green direct distal to the gene. In normal cells you will see two yellow signals (red/green), but in this case there is only one normal yellow signal and a split red and green signal (marked with arrows). Thus there is a translocation affecting the $22 q 12.1-q 12.2$ (the EWSRI gene).

and CD56, while chromogranin was negative. Furthermore, there was no positive stain in muscle or lymphoid markers. The proliferation index was fluctuating from $20 \%$ to $60 \%$, focally higher. Fluorescence In Situ Hybridization (FISH) showed an EWSR1 gene rearrangement (Vysis LSI EWSR1 (22q12) Dual Color, Break Apart Rearrangement Probe) (Figure 2B). Hence, the conclusion of the histopathological examination of the tumor was Ewing sarcoma.

\section{Oncology}

Intradural extramedullary Ewing sarcoma is associated with a poor prognosis with less than $60 \%$ two-year event free survival (EFS) in patients treated with multimodal therapy. Distant craniospinal axis failure is the predominant event in patients treated with focal radiotherapy. ${ }^{2}$

The patient was treated according to the EURO EWING-99 protocol with 6 courses of vincristine, ifosfamide, doxorubicin, and etoposide (VIDE). Four weeks following the VIDE course, the patient started photon irradiation therapy against the entire brain and spinal cord with 36 Gy and a boost to the lumbar spine to a local total dose of 50.4 Gy. Concomitant with the radiation, the patient received one course with vincristine and ifosfamide and one course of vincristine and cyclophosphamide. Following radiation, the patient received and additional three courses of vincristine, actinomycin, and ifosfamide (VAI) and three courses of vincristine, actinomycin, and cyclophosphamide (VAC). The cumulated doses of chemotherapy per $\mathrm{m}^{2}$ were; vincristine $(14 \mathrm{mg}$ ), ifosfamide $(75,360 \mathrm{mg})$, doxorubicin $(320.8 \mathrm{mg})$, etoposide $(2557.5 \mathrm{mg})$, actinomycin $(8.97 \mathrm{mg})$, and cyclophosphamide $(6015 \mathrm{mg})$.

\section{Outcome}

After a seventeen-month treatment-course including highly specialized care at the department of neurosurgery and oncology, the patient is doing well and suffers no sequelae apart from hypogonadism. Imaging 36-month post-surgery reveals no signs of residue or recurrence of pathology in the lumbar spine area.

\section{Discussion}

Primary care family physicians are the gatekeepers to the healthcare system in several European countries including Denmark. ${ }^{3}$ Patients presenting with lumbar spinal pain in family practice are common as back pain is associated with the highest consultation-rate yearly. ${ }^{1,4}$ Furthermore, spinal pain is one of the most common musculoskeletal complaints among care-seeking 14-17-years-olds in primary care family practice. ${ }^{5}$ Although rare, adolescent patients with sinister lumbar spine pathology do present themselves in clinical practice, as demonstrated by the current brief report. Therefore, it is imperative that primary care physicians and additional healthcare professionals remain vigilant towards detecting sinister pathology among their patients. 
Compared to stand-alone radiotherapy ( $10 \%$ overall survival over 5 years) a multimodal treatment approach of Ewing sarcoma consisting of surgery, chemotherapy and/or radiography seems to increase the overall survival significantly (55$65 \%$ overall survival). ${ }^{6,7}$ Early diagnosis and treatment are vital as the presence of metastasis and a primary tumor size of more than $8 \mathrm{~cm}$ in diameter is associated with poor overall survival. ${ }^{6}$ In addition, primary tumors located in the axial skeleton, especially in the pelvic area, are associated with poor overall survival compared to tumors located in the extremities. $^{6}$

This is only the 6th documented case of adolescent intradural extramedullary Ewing sarcoma in the published literature. ${ }^{8}$ As such, not only was it rare for the patient to have an underlying sinister pathology, but the pathology itself was in addition extremely rare. As the patient did not respond to conservative treatment or analgesics, the patient was referred to secondary care diagnostic imaging under the assumption that the imaging findings would change the management of her condition, which reflects current guideline recommendations. ${ }^{9,10}$ Subsequently, the patients received an efficient and timely multimodal treatment, and after three years, the patient was in good health with no signs of residual tumor or recurrence of pathology.

\section{Informed Consent}

Informed consent was obtained from all individual participants included in the study.

\section{Author Contributions}

All authors made a significant contribution to the work reported, whether that is in the conception, study design, execution, acquisition of data, analysis and interpretation, or in all these areas; took part in drafting, revising or critically reviewing the article; gave final approval of the version to be published; have agreed on the journal to which the article has been submitted; and agree to be accountable for all aspects of the work.

\section{Funding}

No funding was received for this research.

\section{Disclosure}

All authors declare no conflicts of interest.

\section{References}

1. Hartvigsen J, Hancock MJ, Kongsted A, et al. What low back pain is and why we need to pay attention. Lancet. 2018;391(10137):2356-2367. doi:10.1016/S0140-6736(18)30480-X

2. Lu VM, Goyal A, Alvi MA, Kerezoudis P, Haddock MG, Bydon M. Primary intradural Ewing's sarcoma of the spine: a systematic review of the literature. Clin Neurol Neurosurg. 2019;177:12-19. doi:10.1016/j.clineuro.2018.12.011

3. Pedersen KM, Andersen JS, Søndergaard J. General practice and primary health care in Denmark. J Am Board Fam Med. $2012 ; 25$ (Suppl 1):34. doi:10.3122/jabfm.2012.02.110216

4. Chew-Graham C, May C. Chronic low back pain in general practice: the challenge of the consultation. Fam Pract. 1999;16(1):46-49. doi:10.1093/ fampra/16.1.46

5. Tan A, Strauss VY, Protheroe J, Dunn KM. Epidemiology of paediatric presentations with musculoskeletal problems in primary care. $B M C$ Musculoskelet Disord. 2018;19(1):40. doi:10.1186/s12891-018-1952-7

6. Bosma SE, Ayu O, Fiocco M, Gelderblom H, Dijkstra PDS. Prognostic factors for survival in Ewing sarcoma: a systematic review. Surg Oncol. 2018;27(4):603-610. doi:10.1016/j.suronc.2018.07.016

7. Werier J, Yao X, Caudrelier J, et al. A systematic review of optimal treatment strategies for localized Ewing's sarcoma of bone after neo-adjuvant chemotherapy. Surg Oncol. 2016;25(1):16-23. doi:10.1016/j.suronc.2015.11.002

8. Scantland JT, Gondim MJ, Koivuniemi AS, Fulkerson DH, Shih C. Primary spinal intradural extraosseous Ewing sarcoma in a pediatric patient: case report and review of the literature. Pediatr Neurosurg. 2018;53(4):222-228. doi:10.1159/000488767

9. Oliveira CB, Maher CG, Pinto RZ, et al. Clinical practice guidelines for the management of non-specific low back pain in primary care: an updated overview. Eur Spine J. 2018;27(11):2791-2803. doi:10.1007/s00586-018-5673-2

10. Foster NE, Anema JR, Cherkin D, et al. Prevention and treatment of low back pain: evidence, challenges, and promising directions. Lancet. 2018;391(10137):2368-2383. doi:10.1016/S0140-6736(18)30489-6 


\section{Publish your work in this journal}

Adolescent Health, Medicine and Therapeutics is an international, peer-reviewed, open access journal focusing on health, pathology, and treatment issues specific to the adolescent age group. All aspects of health maintenance, preventative measures and disease treatment interventions are addressed within the journal and practitioners from all disciplines are invited to submit their work as well as healthcare researchers and patient support groups. The manuscript management system is completely online and includes a very quick and fair peer-review system. Visit http://www.dovepress.com/testimonials.php to read real quotes from published authors.

Submit your manuscript here: http://www.dovepress.com/adolescent-health-medicine-and-therapeutics-journal 